\title{
Study on Reflective Characters of Aircraft Skin Coating Based on Fiber End - face Echoes
}

\author{
Xiang-Lin Zhan, Wei Zhang, Jing-Chang Zhuge, Shu-Jian Xing \\ Deptartment of Electronics, \\ College of Electronic Information \& Automation, \\ Civil Aviation University of China \\ Tianjin, China \\ E-mail: xlzhan@cauc.edu.cn
}

\begin{abstract}
Icing on aircraft skin brings a serious risk to aircraft safety. At present, removing ice is usually conducted by spraying deicing fluid. Even so, the ice on the skin cannot be eliminated completely. Therefore, it is needed to find a fast and accurate detection method to detect the location and area of the residual ice on the aircraft skin. Among numerous nondestructive testing methods, optical fiber detection is an effective method. However, the impact of aircraft coatings has not been considered by the existing fiber detection methods. In fact, coatings have great influences on the icing detection result if optical sensors are used. To solve this problem, a fiber endface echo model based on the dual-flux theory is established, and the reflection characteristics of coatings on aircraft skin are studied in this article. The detection principle of aircraft residual icing based on optical fibers and the model are introduced. Characteristics of aircraft skin coatings and possible impacts on detection of residual ice are described. The reflection law of the fiber end face is discussed. The experiments are carried out and the obtained reflection law of different color painting is validated. The impact of aircraft skin coatings on the aircraft residual ice detection can be effectively eliminated, and the detection accuracy can be improved.
\end{abstract}

Keywords-fiber optical sensor; aircraft skin; coatings; reflection characteristic

\section{INTRODUCTION}

Civil aviation aircrafts are easy to be frozen in the winter. If a plane takes off with ice on its wings, the aircraft aerodynamic force will be affected seriously, causing the airplane stalled. So, the possible presence of residual ice on an aircraft wing is a great hazard to flight safety. According to the statistics provided by the US National Transportation Safety Board, 82\% of accidents were caused by aircraft icing or frost when the aircraft took off, among which $70 \%$ are fatal disasters [1]. Therefore, deicing and residual icing detection are very important. In the winter after preflight deicing operations, it is required to detect the residual ice before a plane takes off [2-5]. Currently, the widespread detection methods are still manual visual and tactile detection. Their detection efficiency is very low. The safety and efficient operation of the civil aviation in the winter cannot be ensured. As one of the important facilities of the civil aviation airport security in our country, the residual ice detection technology is needed to be improved, which is of great significance to promote the healthy development of China's civil aviation.

A variety of methods have been studied for automated ice detection of aircraft de-icing, but most of them are at the experimental stage. A laser scanning method was developed by Aerospace Goodrich Company in the United States [6]. A linear polarized laser source was used to scan the surface of the plane, and the received reflection laser was used to determine the residual ice. The method can detect the residual ice, but it needs to be scanned point by point, and the detection efficiency is not high. MDA company developed the ROGIDS residual ice detection system, which is based on the infrared multi-spectral imaging principle [7]. It can detect the shape and thickness of the ice. Huazhong University of Science and Technology has successfully developed an optical fiber sensor and film sensors, one of which can also identify the ice type [8]. A residual ice detection method based on near infrared multi spectrum has been proposed by Tianjin University [9]. The thickness detection accuracy can reach to $0.5 \mathrm{~mm}$. Among numerous residual ice detection methods, the fiber optic icing sensor is more competitive because of its small size, antielectroma1gnetic interference. Civil aviation aircraft skin surface is usually painted in various colors of the undercoat and the topcoat, to meet airworthiness requirements and aesthetic effects. The optical detection results are affected by these coating materials and colors, whose influence cannot be ignored. Current detection methods of the aircraft residual ice do not consider the impact of aircraft skin surface coatings. It will inevitably lead to false detection. Therefore, it is the key to improve the detection technology of residual ice and find out the influences of aircraft skin coating in advance.

\section{THE DETECTION PRINCIPLE OF RESIDUAL ICING}

\section{A. The Basic Principle of Optical Fiber Sensor}

In this article, optical fiber sensors are used. Based on the principle of reflection and scattering of light, the light emitted by the light source is transmitted to the detected surface through an optical fiber. When the ice or snow is covered on the detected area, the receiving optical fiber sensor cannot receive the reflected light because all light is scattered into the air. If the detection surface is attached by 
the residual ice, a series of optical effects occur in the ice, such as scattering, reflection and transmission. The light scattered in the ice and reflected at the interface can be collected by the receiving fiber, as shown in Fig. 1.

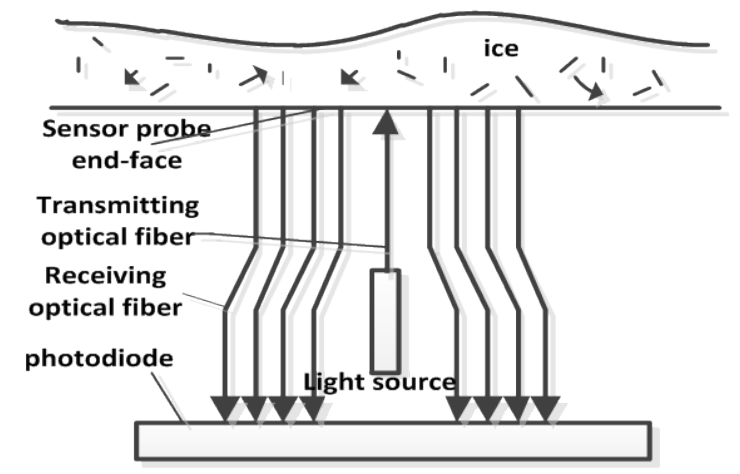

Figure 1. Schematic of an Optical fiber sensor.

Surfaces in Fig.1 are all supposed to be smooth but are made of different materials. As materials have different reflecting and scattering abilities, the light intensities received by the receiving fiber are different. Through the photoelectric detector and the signal conditioning circuit, the strength of the output electrical signal corresponds to the intensity of the input optical signal. Then, the residual ice can be detected by processing the electric signal.

\section{B. Analysis of Rreflection Character for Optical Fiber End Face}

When the light is reflected from the airplane surface into the optical fiber end face, it comes from the air medium to the optical fiber medium. There are many problems. The propagation direction of the light changes, distribution of energy varies, and phase transitions and the polarization change too. The refractive index of air and fiber are supposed to be $n_{1}$ and $n_{2}$ respectively. When an incident beam of light comes from the natural air side, it is reflected or refracted before reaching the fiber end, as shown in Fig. 2.

The plane which is composed of the optical fiber end face and the incident light is called the incident plane. The angle between the incident ray and the normal is the incident angle. Similarly, the angle between the reflected ray and the normal is the reflection angle. Also, the angle between the refraction ray and the normal is the refractive angle. If the amplitude of any electric vector in the natural light is $E$, the projection of all the electric vectors that paralleling to the incident plane $E_{X}$ and perpendicular to the incident plane $E_{Y}$ are:

$$
\left\{\begin{array}{l}
E_{X}=\sum E_{i x} \\
E_{Y}=\sum E_{i y}
\end{array}\right.
$$

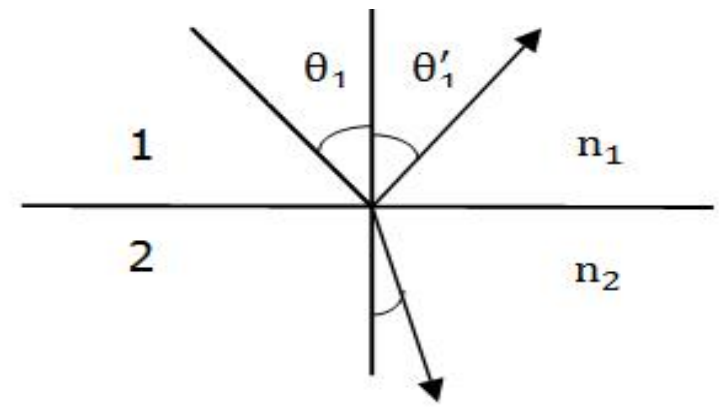

Figure 2. Reflection and refraction model of the optical fiber end face.

The intensity of incident light is:

$$
I=I_{x}+I_{y}
$$

The amplitudes and intensities of the incident light, reflected light and refraction light consist of two components. One that parallels to the incident plane is represented by "p", and the other one that is perpendicular to the incident plane is represented by "s". Based on the Fresnel formula, the reflection and refraction amplitudes can be indicated by (3):

$$
\left\{\begin{array}{l}
E_{1 p}^{\prime}=\frac{n_{2} \cos \theta_{1}-n_{1} \cos \theta_{2}}{n_{2} \cos \theta_{1}+n_{1} \cos \theta_{2}} E_{1 P}=\frac{\tan \left(\theta_{1}-\theta_{2}\right)}{\tan \left(\theta_{1}+\theta_{2}\right)} E_{1 p} \\
E_{2 p}=\frac{2 n_{1} \cos \theta_{1}}{n_{2} \cos \theta_{1}+n_{1} \cos \theta_{2}} E_{1 p} \\
E_{1 s}^{\prime}=\frac{n_{1} \cos \theta_{1}-n_{2} \cos \theta_{2}}{n_{1} \cos \theta_{1}+n_{2} \cos \theta_{2}} E_{1 s}=\frac{\sin \left(\theta_{2}-\theta_{1}\right)}{\sin \left(\theta_{1}+\theta_{2}\right)} E_{1 s} \\
E_{2 s}=\frac{2 n_{1} \cos \theta_{1}}{n_{1} \cos \theta_{1}+n_{2} \cos \theta_{2}} E_{1 s}=\frac{2 \cos \theta_{1} \sin \theta_{2}}{\sin \left(\theta_{1}+\theta_{2}\right)} E_{1 s}
\end{array}\right.
$$

In (3), each field component can be understood both as an instantaneous value and as a complex vibration value, because they have the same time frequency. The Fresnel formula shows that the p component of the reflected and refracted light is only associated with the p component in the incident light, and the $\mathrm{s}$ component is only related to the $\mathrm{s}$ component. That is, in the reflection and refraction process, $\mathrm{p}$ and s components of vibration are independent of each other. According to the Fresnel formula (3), the intensity components can be obtained: 


$$
\left\{\begin{array}{l}
I_{1 p}^{\prime}=\left(E_{1 P}^{\prime}\right)^{2}=\left(\frac{\tan \left(\theta_{1}-\theta_{2}\right)}{\tan \left(\theta_{1}+\theta_{2}\right)}\right)^{2} I_{1 p} \\
I_{2 p}=\left(E_{2 p}\right)^{2}=\left(\frac{2 m_{1} \cos \theta_{1}}{n_{2} \cos \theta_{1}+n_{1} \cos \theta_{2}}\right)^{2} I_{1 p} \\
I_{1 s}^{\prime}=\left(E_{1 s}^{\prime}\right)^{2}=\left(\frac{\sin \left(\theta_{2}-\theta_{1}\right)}{\sin \left(\theta_{1}+\theta_{2}\right)}\right)^{2} I_{1 s} \\
I_{2 s}=\left(E_{2 s}\right)^{2}=\left(\frac{2 \cos \theta_{1} \sin \theta_{2}}{\sin \left(\theta_{1}+\theta_{2}\right)}\right) I_{1 s}
\end{array}\right.
$$

Therefore, the light intensity can be got:

$$
I_{1}{ }^{\prime}=I_{1 p}{ }^{\prime}+I_{1 s}{ }^{\prime} .
$$

The intensity component of parallel direction is the same to that of the vertical direction:

$$
I_{1 s}=I_{1 p}, \quad I_{1}=I_{1 p}+I_{1 s} .
$$

Then, (5) can be computed as:

$$
\begin{aligned}
& I_{1}^{\prime}=I_{1 p}^{\prime}+I_{1 s}^{\prime} \\
& =\left(\frac{\tan \left(\theta_{1}-\theta_{2}\right)}{\tan \left(\theta_{1}+\theta_{2}\right)}\right)^{2} I_{1 p}+\left(\frac{\sin \left(\theta_{2}-\theta_{1}\right)}{\sin \left(\theta_{1}+\theta_{2}\right)}\right)^{2} I_{1 s}
\end{aligned}
$$

So the light intensity reflectivity $R$ is:

$$
R=\frac{I_{1}^{\prime}}{I_{1}}=\frac{1}{2}\left[\left(\frac{\tan \left(\theta_{1}-\theta_{2}\right)}{\tan \left(\theta_{1}+\theta_{2}\right)}\right)^{2}+\left(\frac{\sin \left(\theta_{2}-\theta_{1}\right)}{\sin \left(\theta_{1}+\theta_{2}\right)}\right)^{2}\right]
$$

When the incident angle is small or close to $0, R$ can be simplified as:

$$
R=\frac{I_{1}^{\prime}}{I_{1}}=\left(\frac{n_{2}-n_{1}}{n_{2}+n_{1}}\right)^{2}
$$

From the above analysis, the reflectivity of light at the end face of the optical fiber is obtained. It can be seen that the reflectivity of the end face of the fiber depends on the angle of the incident light, and is related to the refractivity the air and fiber material. In this article, the end face of the optical fiber sensor is perpendicular to the surface of the aircraft skin, the incident angles are the same. Only the refractivity of the material is discussed here.

\section{ANALYSIS OF COATING CHARACTERISTICS OF AIRCRAFT SKIN}

Aircraft skin coating can be used as the identification material of the aircraft outer surface, playing the role of anticorrosion, decoration and so on [13]. Even it has special functions of temperature mark, anti-skid, damping and absorbing. Civil aircraft skin coating has an important anticorrosion function, which makes the aircraft maintain good performance in high altitude harsh environmental conditions. Aircraft coating includes two materials: the undercoat and the topcoat. Polyurethane undercoat and polyurethane topcoat are mainly used for Airbus aircraft skin, while epoxy undercoat and polyurethane topcoat are mostly used for Boeing aircraft skin [14]. When there is residual ice on the aircraft surface, the ice directly contacts with the coating layer of the aircraft skin and then the base material. So, the reflection characteristic of the aircraft skin coating has a great influence and cannot be neglected.

To solve this problem, the phenomenological theory is put forward to. This theory proposed that the coating layer of the aircraft skin surface can be regarded as a layer of film. The light energy is processed without considering the coating particles attributes. Applying the film's reflection, scattering and absorption principle, when the light is incident on an incomplete transparent surface, four cases will occur as shown in Fig. 3:

(1) There is a specular reflection on the smooth surface, and a diffuse reflection at the rough surface at the same time;

(2) The light is absorbed or scattered by particles in the film after it enters the film;

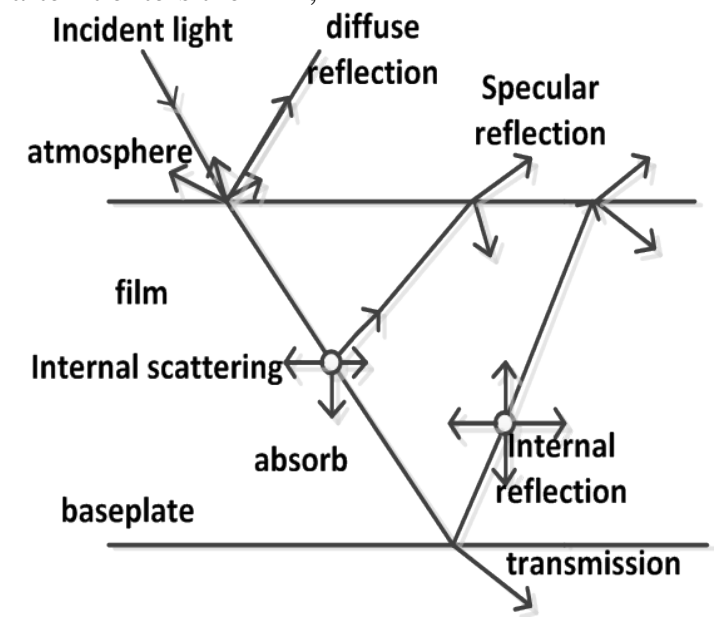

Figure 3. Propagation of light in a thin film layer.

(3) Part of the light is reflected and others is transmitted through the film layer when the light reaches the bottom part of the film;

(4) The light that does not leave the film continues the above cycle.

The propagation of light in the thin-film layer is very complex, but the Kubelka-Munk dual-flux theory can well explain it. According to the theory, a fiber end-face echo model can be established. The aircraft skin coating is regarded as a layer of film, its reflectance $\rho$ can be 
characterized by color, representing the light absorption and reflection properties of coating materials. It can be expressed as:

$$
\rho=\frac{1-\rho_{g}[a-b \operatorname{coth}(b S X)]}{a-\rho_{g}+b \operatorname{coth}(b S X)}
$$

where $a=1+(K+S), b=\left(a^{2}-1\right)^{1 / 2}, \operatorname{Coth}(b S X)$ is a hyperbolic cotangent function, $K$ is the absorption coefficient, $S$ is the scattering coefficient, $X$ is the film thickness and $\rho_{g}$ is the basal reflection ratio.

Equation (10) is the basic equation of the Kubelka-Munk theory, from which we can see that the reflection ratio of semi-transparent or opaque medium is a function of $K, S, X$ and $\rho_{g}$. It can be referred that the reflection ratio $\rho$ is influenced by the absorption and scattering coefficients of the film as well as the reflection coefficient of the film and the substrate.

Therefore, based on the model, the light absorption and reflection properties of coating vary according to its color and material. Usually, green corrosion-resistant undercoat is spayed on the aircraft. However, some parts of the aircraft have no undercoat. There are many kinds of topcoat used for the aircraft. They have different reflectivity. Therefore, experiments are carried out on a test block with green undercoat and a block without undercoat.

\section{EXPERIMENTS}

Aluminum alloy material has been widely used as aircraft body materials, because it has the advantages of light weight, high speed, high effective load and strong corrosion resistance and so on. In the laboratory environment, an aluminum alloy board is supposed to be the aircraft skin, the light reflection of different colors of paint is converted into an electrical signal through the optical fiber sensor. Then, different color coating reflection characteristics are analyzed. In this experiment, aluminum alloy 2024 was used as the aircraft skin substrate. On one side of the substrate, five colors of topcoat BMS10-60I protective enamel were directly sprayed. The colors are red, black, white, gray and blue, as shown in Fig. 4 (a). On the other side, BMS10-11I green corrosion-resistant undercoat was sprayed, and then the above five colors of topcoat were sprayed, as shown in Fig. 4 (b). This is one of coating programs for Boeing aircrafts. It is carried out by special equipment for spraying operations in aircraft maintenance manufacturers, to ensure uniform coating and close to the actual aircraft skin coating state.

The fiber optic sensor probe was placed to face different colors. Ans the light source was perpendicular to the sensor, which got the returned signals. The maximum and minimum values of each signal data are removed, and the rest of the data are averaged, as shown in Table 1.

TABLE I. AVERAGE AMPLITUDE VALUES OF RETURNED SIGNALS FOR DIFFERENT COLOR SURFACES

\begin{tabular}{|c|c|c|}
\hline Color & Without Undercoat (V) & $\begin{array}{c}\text { With Undercoat } \\
\text { (V) }\end{array}$ \\
\hline $\begin{array}{c}\text { only green } \\
\text { undercoat }\end{array}$ & 1.602 & 1.630 \\
\hline Red & 1.612 & 1.628 \\
\hline Black & 1.588 & 1.596 \\
\hline Gray & 1.600 & 1.612 \\
\hline White & 1.628 & 1.663 \\
\hline Blue & 1.592 & 1.618 \\
\hline
\end{tabular}

Based on Table 1, the relationship between the color of the paint and returned voltage signals are drawn in Fig. 5. The histogram is shown as Fig. 6.

From the above experimental data, we can learn that the returned signal values from the board with undercoat are all larger than those from the board without undercoat. Furthermore, the return signal values of each color are not the same. Therefore, the influences of aircraft skin coating material and color on the detection result cannot be ignored. The return values of different coating color are listed in Table 2 in order.

TABLE II. RETURNED SIGNAL VALUES OF DIFFERENT COATING COLORS

\begin{tabular}{|c|c|c|}
\hline \multirow{2}{*}{$\begin{array}{c}\text { Return } \\
\text { signal } \\
\text { value } \\
\text { From } \\
\begin{array}{c}\text { Small } \\
\text { to } \\
\text { Large } \\
\text { Order }\end{array}\end{array}$} & Black & With Undercoat \\
\cline { 2 - 3 } & Blue & Black \\
\cline { 2 - 3 } & Baseboard & Blue \\
\cline { 2 - 3 } & Gray & Red \\
\cline { 2 - 3 } & White & only primer \\
\hline
\end{tabular}

From Table 2, a rule can be found from the values of different colors of coatings. The amplitude value of Black is the lowest whereas that of White is the highest. An initial judgement the coating color can be determined. In the actual residual ice detection, according to the above different reflection characteristics of the coating color, the returned signal values can be corrected. It can reduce the impact of coating color, and improve the detection efficiency of residual ice, which can greatly reduce the probability of false detection and missed detection. 


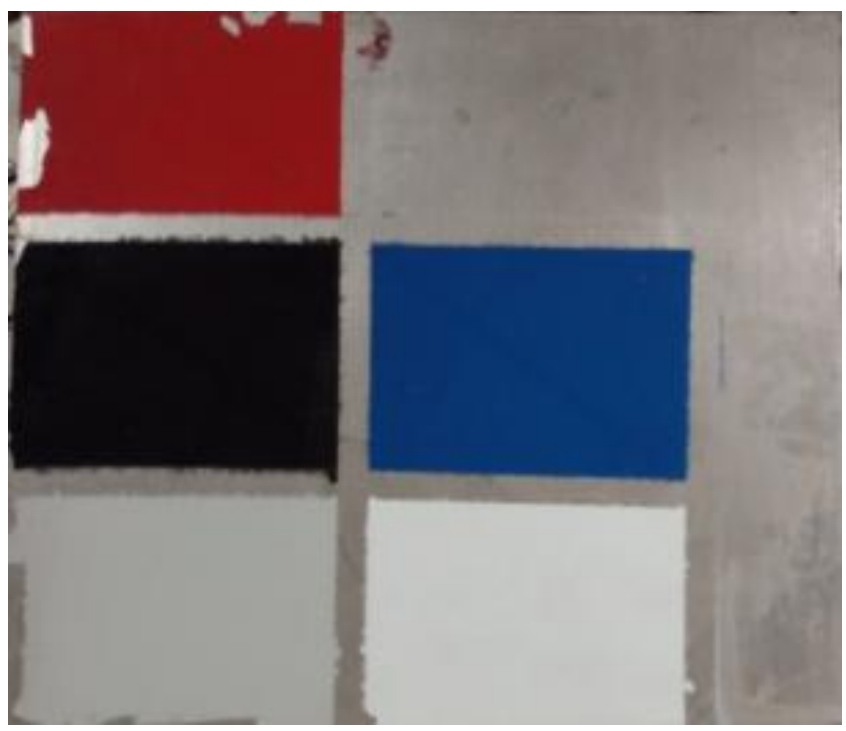

(a).Five colors of topcoat sprayed on the aluminum board

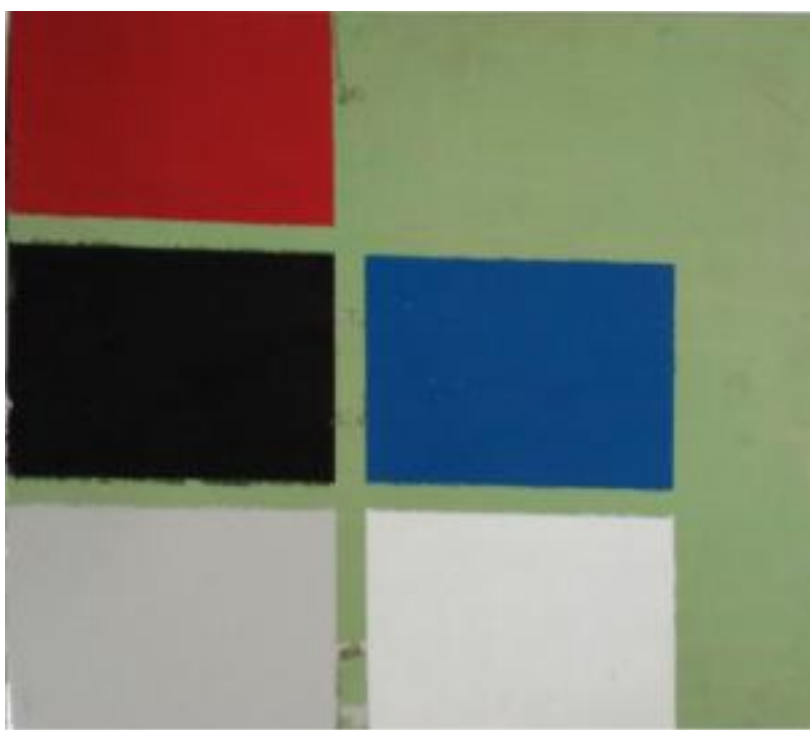

(b) Five colors of topcoat sprayed on the green undercoat

Figure 4. Double sides of an aluminum alloy board sprayed with coatings.

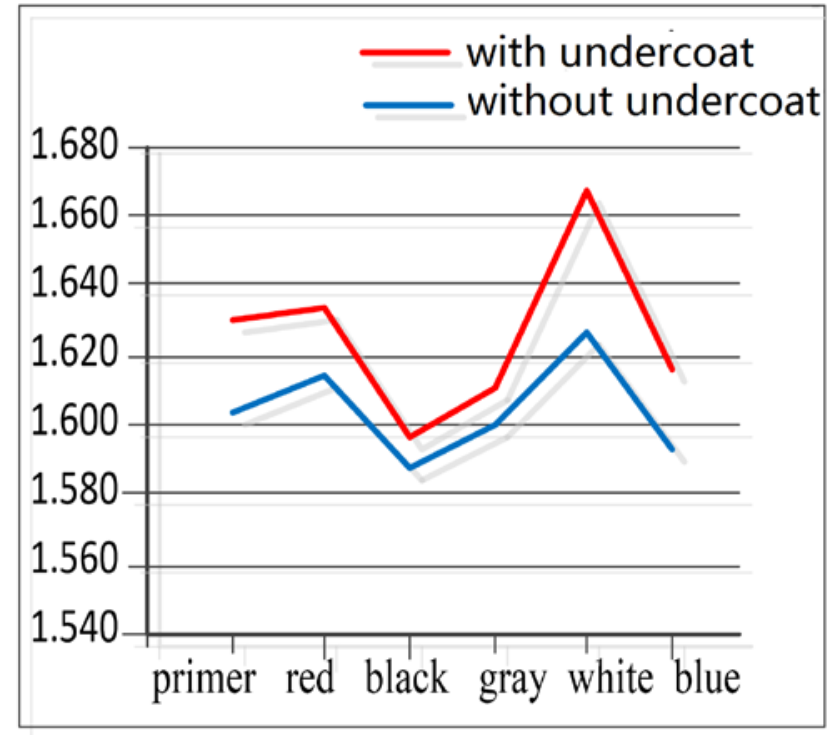

Figure 5. Schematic diagram of the relationship between the paint color and returned voltage signals

\section{CONCLUSIONS}

In this article, the reflection characteristics of aircraft skin coating based on the end-echo are studied. The influence of the optical fiber sensor on the detection of the residual ice on is discussed. Experiments show that the impact of aircraft skin coating cannot be ignored, and a rule exists between the color of aircraft skin coating and the signal values of the fiber optic sensor. In the actual ice detection, no matter what the color of the plane surface coating is, we can advance the test to record signal values corresponding to the coating color, and to establish a database to preserve the detection results. In this way, when

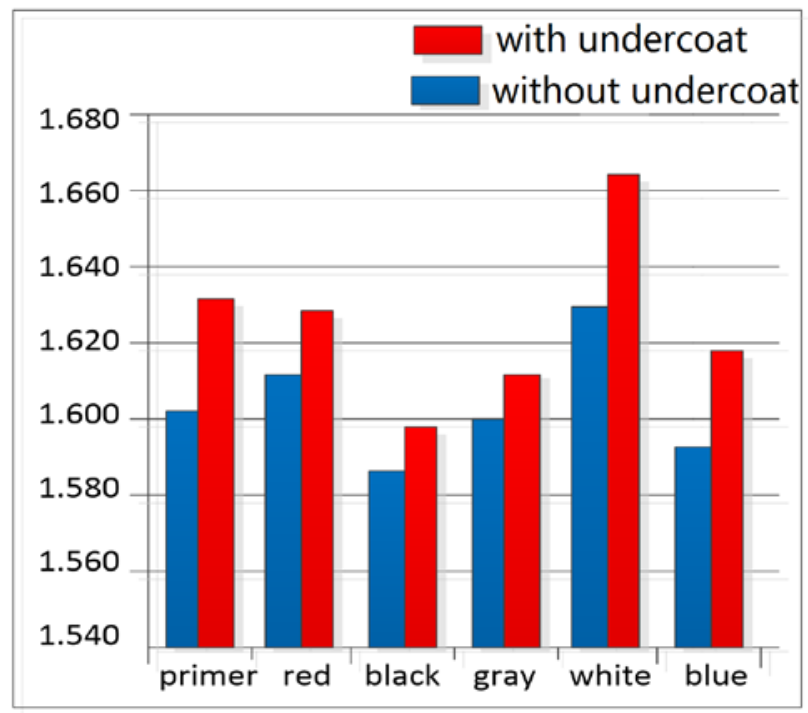

Figure 6. Histogram of the relationship between the paint color and returned voltage signals

the inspection is conducted, the detected signal of residual ice can be corrected by the database, in order to improve the detection accuracy.

\section{ACKNOWLEDGMENT}

The authors are very grateful for the support of NSFC (Grant NO. 61405246 \& NO. U1533111) and fund of CAUC(Grant NO. 3122015C012 \& NO. 2014QD11X)

\section{REFERENCES}

[1] Xie Yi, Statistical analysis of aircraft icing accidents [J], Foreign test, (3):23-28, 1996. 
[2] I. B. Vermeulen, S.M. Bohte, and D.J.A. Somefun, Improving patient schedules by multi-agent pareto appointment exchanging[C], Proceedings of 2006 IEEE International Conference on E-Commerce Technology (CEC/EEE2006), San Francisco, California, June 26-29, 2006.

[3] Xiaoyu Mao, Adriaan ter Mors, Nico Roos, and Cees Witteveen, Coordinating Competitive Agents in Dynamic Airport Resource Scheduling[C], Proceedings of the Fifth German Conference on Multiagent System Technologies (MATES2007), Lecture Notes in Artificial Intelligence 4687, Springer, 2007.

[4] Transportation Development Centre, Tactile Inspection for Detection of Ice on Aircraft Surfaces. Transport Canada Report, TP 13858E, 2004.

[5] Transportation Development Centre, Civil Aviation Transport Canada, Federal Aviation Administration, Development of Ice Samples for Comparison Study of Human and Sensor Capability to Detect Ice on Aircraft. Transport Canada Report, TP 14450E, 2006.

[6] C Narlis, Development of Ice Samples for Comparison Study of Human and Sensor Capability to Detect Ice on Aircraft[J], Deicing, (1): 72-76, 2006.

[7] Scott M. Terrace, Kimberlea D. Bender, Edmundo. Sierra, Comparison of Human Ice Detection Capabilities and Ground Ice Detection System Performance Under Post Deicing Conditions, Human Factors \& Ergonomics Society Annual Meeting Proceedings, 50(17):2051-2055, 2006.
[8] Yin Shengsheng, YE Lin, CHEN Bin, GE Jun-feng, and WANG Liwen, Fiber-optical Icing Sensor for Detecting the Icing Type [J], Instrument Technique and Sensor, (05):9-12, 2012.

[9] GAO Jian-shu, HAN Ren-yi, YU Zhi-jing, QIAO Wen, Composite wing surface ice near infrared multispectral detection [J]. Optics and precision engineering, 19(6):1250-1255, 2011.

[10] Sun Peng, Jiang Liangguang. A model of the relationship between the refractive index of water and the wavelength of the water $[\mathrm{J}]$. Spectrum laboratory,19(4):554-556, 2002.

[11] Wenfeng, Luo Zhiquan. Energy distribution of natural light in the process of reflection and refraction [J]. Journal of China West Normal University (natural science edition), 29(02) :208-211, 2008.

[12] Roger N Clark, Water Frost and ice: The near-infrared spectral reflectance 0.65-2.5um [J]. Journal of geophysical research, 86(B4):3087-3096, 1981.

[13] Zhao Xin, Xie Fei, Zhang Fan, Anti-corrosion system of modern civil aviation aircraft skin[J], Total corrosion control, 28(1):22-24, 2014.

[14] Adriaan ter Mors, Xiaoyu Mao, Multi-Agent System Support for Scheduling Aircraft Deicing[C], Proceeding of ISCRAM 2007 Intelligent Human Computer Systems for Crisis Response and Management, VUBPRESS Brussels University Press, 2007.

[15] Zhao Xin, Yu Xinhua, Su Zhengliang, Analysis of test standards and airwothiness requirements for exterior decorative coating systems of civil aviation aircrafts[J], Paint \& Coatings Industry, 44(8): 69-74, 2014. 